\title{
Vigilancia de la resistencia del Mycobacterium tuberculosis a los fármacos antituberculosos, Colombia 2004-2005
}

\author{
María Consuelo Garzón¹, Dailyn Yorledy Angée, Claudia Llerena1, Dora Leticia Orjuela", \\ Jorge Ernesto Victoria ${ }^{2}$. \\ ${ }^{1}$ Grupo de Micobacterias, Instituto Nacional de Salud, Bogotá D.C. Colombia \\ ${ }^{2}$ Organización Panamericana de la Salud, Representación Colombia
}

Introducción. La tuberculosis es una importante causa de enfermedad y muerte en el mundo. Se calcula que en el 2005 se presentaron 8,8 millones de casos nuevos y murieron 1,6 millones de personas, entre ellas 195.000 infectadas con VIH. Según la Organización Mundial de la Salud, la tasa de incidencia permaneció estable o disminuyó en todo el mundo; sin embargo, el número de casos nuevos se incrementó debido al aumento en algunas regiones.

La resistencia a los medicamentos es un problema de salud pública y un obstáculo al control de la tuberculosis en el mundo. Por esta razón, es necesario generar medidas para el adecuado manejo de los pacientes y adoptar estrategias para prevenir su diseminación.

Objetivo. Determinar la prevalencia de la resistencia de Mycobacterium tuberculosis tanto en casos no tratados como en casos previamente tratados de tuberculosis pulmonar en Colombia. Materiales y métodos. Estudio de corte transversal para determinar la prevalencia de la resistencia de $M$. tuberculosis a fármacos antituberculosos en 1.189 pacientes durante los años 2004 y 2005. La recolección de cultivos fue a nivel nacional durante 1 año; los aislamientos se procesaron para pruebas de susceptibilidad por la técnica de proporciones múltiples de Cannetti, Risk y Grooset en su variante simplificada.

Resultados. Novecientos veinticinco pacientes no tratados presentaron una prevalencia de resistencia global de 11,78\% (IC 95\%: 9,86-14,02) y una tuberculosis multirresistente (multidrugresistant tuberculosis, MDR-TB) de 2,38\% (IC 95\%: 1,58-3,57). Los 264 pacientes previamente tratados presentaron una resistencia global de $44,32 \%$ (IC 95\%: $38,45-50,35$ ) y una tuberculosis multirresistente (MDR-TB) de 31,44\% (IC 95\%: 26,14-37,27).

Conclusiones. Los resultados obtenidos, comparados con estudios previos, demuestran que no ha habido un aumento significativo en la resistencia a los medicamentos ni en la tuberculosis multirresistente. Los hallazgos indican que el esquema proporcionado por el Programa Nacional de Tuberculosis es adecuado para manejar los casos.

Palabras clave: tuberculosis, tuberculosis resistente a múltiples medicamentos, Mycobacterium tuberculosis, susceptibilidad, resistencia a los medicamentos

\section{Surveillance of Mycobacterium tuberculosis resistance to antituberculosis drugs}

Introduction. Tuberculosis is an important cause of disease and death worldwide. An estimated 8.8 million new cases occurred in 2005 with 1.6 million deaths, including 195,000 among HIVinfected people. According to World Health Organization, the incidence rate was stable or in decline worldwide; however, the total number of new cases rose due to regional increases. Anti-TB drug resistance is a significant public health problem and an obstacle for its control worldwide. Therefore, measures must be taken for the adequate management of patients and the adoption of strategies to prevent TB dissemination.

Objective. The prevalence of resistance of Mycobacterium tuberculosis was determined in untreated cases and in previously treated cases of pulmonary tuberculosis in Colombia.

Materials and methods. A cross-sectional study determined the prevalence of resistance of Mycobacterium tuberculosis to antituberculosis drugs in 1,189 untreated cases or previously treated cases of pulmonary tuberculosis between the years 2004 and 2005. Cultures were collected throughout the country for this one-year period. Drug susceptibility of the isolates was 
tested by the simplified variant of the Cannetti, Risk and Grooset multiple proportions technique. Results. The global resistance rate of 925 untreated patients was $11.8 \%$ (95\% Cl: $9-14 \%)$ and the rate of multidrug-resistant tuberculosis was $2.4 \%(95 \% \mathrm{Cl}: 1.6-3.6 \%)$. Among 264 previously treated patients, the rate of global resistance was $44.3 \%(95 \% \mathrm{Cl}: 38-50 \%)$ and that of multidrug resistance was $31.4 \%(95 \% \mathrm{Cl}: 26-37 \%)$.

Conclusions. When compared to previous studies, these data show that there has not been a significant increase in drug resistance. The findings indicate that the current treatment scheme provided by the National Tuberculosis Program is adequate.

Key words. Tuberculosis, multidrug-resistant tuberculosis, Mycobacterium tuberculosis, drug resistance, susceptibility.

La tuberculosis sigue siendo una importante causa de enfermedad y muerte en todo el mundo. Se calcula que en el 2005 hubo 8,8 millones de casos nuevos, de los cuales, 7,4 millones se presentaron en Asia y África subsahariana, y causó la muerte de 1,6 millones de personas, entre ellas 195.000 infectadas con el VIH (1).

Las tasas mundiales de prevalencia y mortalidad de la tuberculosis, probablemente, han estado en descenso durante varios años. Según la Organización Mundial de la Salud (OMS), la tasa de incidencia permaneció estable o disminuyó en todo el mundo. Sin embargo, el número absoluto de casos nuevos se incrementó, debido al aumento en las regiones de África, Mediterráneo oriental y Asia suroriental (1).

La aparición de la resistencia a los fármacos antituberculosos y, en particular, la tuberculosis resistente a múltiples medicamentos (multidrugresistant tuberculosis, MDR-TB) -definida como resistencia, por lo menos, a rifampicina e isoniacida- se ha convertido en un problema significativo de salud pública en algunos países y en un obstáculo al control eficaz de la tuberculosis en todo el mundo. En muchos países se desconoce el grado de resistencia a los fármacos y es inadecuado el manejo de los enfermos de tuberculosis multirresistente. En los países en donde se ha identificado la resistencia a los fármacos, se deben tomar medidas específicas dentro de los programas de control

\footnotetext{
Correspondencia:

Correspondencia: Claudia Llerena Polo, Grupo de Micobacterias, Instituto Nacional de Salud. Avenida Calle 26 № 51-20.Teléfono: 57-1-2200926 2207700 extensión 436 o 498, fax: 57-1-2200926, cllerena@ins.gov.co

Recibido: 07/09/07; aceptado:02/04/08
}

de la tuberculosis para abordar el problema mediante el manejo apropiado de los pacientes y la adopción de estrategias para prevenir la aparición y la propagación de la tuberculosis resistencia a los medicamentos (2).

Colombia cuenta con una población de 42'090.502 habitantes; la incidencia de tuberculosis ha presentado un descenso en los últimos años, aunque con una tendencia sostenida al incremento a partir de 1997 (3). Para 2005, la tasa de incidencia de todas las formas de tuberculosis fue de 25,2 casos por 100.000 habitantes. El total de casos nuevos de tuberculosis reportados fue de 11.625, de los cuales, 7.706 correspondieron a tuberculosis con baciloscopia positiva (3).

En el país se han realizado dos estudios de resistencia de Mycobacterium tuberculosis a los fármacos antituberculosos en pacientes no tratados. En el primer estudio, realizado en 1992, se incluyeron 829 pacientes, con una resistencia global de $14,1 \%$ y una MDR-TB de 1,81\% (4). En el segundo estudio, adelantado durante los años 1999 y 2000 , se incluyeron 1.087 pacientes con una resistencia global de $15,6 \%$ y una MDR-TB de $1,5 \%$ (5).

La vigilancia de la resistencia a los fármacos antituberculosos es esencial para disponer de información sobre la magnitud y las tendencias de la resistencia a los medicamentos, formular y evaluar directrices de tratamiento y vigilar el efecto de las intervenciones. Por esta razón, el Programa Nacional de Control de Tuberculosis mantiene como estrategia la realización periódica de este tipo de estudios, con el objetivo de determinar la prevalencia de la resistencia en el territorio nacional de casos de tuberculosis pulmonar, previamente tratados y sin tratar. 


\section{Materiales y métodos}

Se llevó a cabo un estudio de corte transversal de vigilancia de la resistencia de $M$. tuberculosis a los fármacos antituberculosos. La recolección de muestras la hizo durante los años 2004 y 2005 la Red Nacional de Laboratorios a través de los laboratorios de salud pública, departamentales y de los distritos, de las secretarías de salud del país.

Pacientes: se incluyeron 1.189 pacientes con tuberculosis pulmonar con baciloscopia positiva, y se dividieron en dos grupos: no tratados y previamente tratados. Para el grupo de pacientes no tratados, se incluyeron los casos nuevos de tuberculosis pulmonar con baciloscopia positiva de esputo, mayores de 15 años, sin antecedentes de tratamiento con uno o más fármacos antituberculosos. Teniendo en cuenta los 7.787 casos de tuberculosis pulmonar con baciloscopia positiva, registrados en el Programa Nacional de Control de Tuberculosis en el año 2002, y los porcentajes de resistencia a los fármacos obtenidos en estudios anteriores, se calculó el tamaño de la muestra para cada departamento, empleando el valor de la prevalencia de resistencia al etambutol, fármaco que presentó el porcentaje de resistencia más bajo en el estudio anterior, y utilizando el programa Epi-Info 6,04.

Con esta información se proyectó el tamaño de muestra, proporcional al número de casos registrados por departamento; el valor obtenido fue de 1.055 pacientes no tratados con un nivel de confianza del $95 \%$ y una precisión del 0,5\%; a este valor se le adicionó el $20 \%$ considerando las probabilidades de pérdida, como cultivos negativos o contaminados y aquéllos cuyas pruebas de susceptibilidad presentaron bajo conteo y no pudieran ser interpretadas. El tamaño obtenido fue de de 1.266 pacientes.

Para facilitar el proceso de recolección de cultivos, se dividió el país en dos grupos: el primero, del $1{ }^{\circ}$ de septiembre de 2004 al 28 de febrero de 2005 , y el segundo, del $1^{\circ}$ de marzo al 30 de agosto de 2005, con un período de seis meses para cada grupo (cuadro 1).

Para el grupo de pacientes previamente tratados se proyectó un tamaño de muestra de 243 casos, basados en la teoría de la OMS según la cual: "la proporción de pacientes previamente tratados suele ser tan sólo una pequeña fracción del total de la población" (6); en este grupo se incluyó el $100 \%$ de los pacientes con historia de fracaso, abandono del tratamiento o recaída, que asistieron a los centros de atención durante un año.

Pruebas de laboratorio: los cultivos se procesaron en el Laboratorio Nacional de Referencia, y se realizó prueba de susceptibilidad de $M$. tuberculosis a los fármacos antituberculosos estreptomicina, isoniacida, rifampicina y etambutol, mediante la técnica de las proporciones múltiples de Canetti, Rist y Grosset en su variante simplificada (7-9).

Control de calidad: durante el desarrollo del estudio se realizó control de calidad a las baciloscopias de diagnóstico, a los medios de cultivo Ogawa Kudoh y Stonebrink modificados por Giraldo preparados por los laboratorios de salud pública, y al medio de Lowenstein Jensen preparado por el Instituto Nacional de Salud, utilizado para las pruebas de susceptibilidad de acuerdo con los lineamientos y metodología estandarizados por el Laboratorio Nacional de Referencia de Micobacterias $(10,11)$.

La calidad de las pruebas de susceptibilidad fue evaluada por el laboratorio supranacional del Instituto de Salud Pública de Chile, del cual se recibieron 60 cepas de referencia para el control directo de calidad y, posteriormente, se enviaron 201 cepas colombianas tanto resistentes como sensibles a los medicamentos para el control indirecto de calidad (12).

El manejo de la información se realizó mediante el programa Surveillance of drug resistance in tuberculosis (SDRTB) (13).

\section{Aspectos éticos}

Todo paciente que ingresó al estudio firmó el consentimiento informado con el que tuvo conocimiento de los procedimientos que se iban a realizar, aclarando que no existía ningún riesgo para el paciente; además, se diligenció un formato de historia que incluía datos individuales, antecedentes clínico-epidemiológicos y de quimioterapia y la información obtenida de la tarjeta individual de tratamiento e historia clínica. 
Cuadro 1. Cumplimiento por departamento de recolección de muestras de los casos no tratados.

\begin{tabular}{|c|c|c|c|c|c|c|c|c|c|c|c|}
\hline \multirow[b]{2}{*}{ Departamento } & \multirow[b]{2}{*}{$\begin{array}{l}\text { Tamaño } \\
\text { muestra }\end{array}$} & \multirow[b]{2}{*}{$\begin{array}{l}\text { Cultivos } \\
\text { recibidos }\end{array}$} & \multicolumn{7}{|c|}{ Cultivos descartados } & \multirow[b]{2}{*}{ Cultivos } & \multirow[b]{2}{*}{$\begin{array}{l}\text { Porcentaje } \\
\text { cumplimiento }\end{array}$} \\
\hline & & & $\begin{array}{c}\text { Bajo } \\
\text { recuento }\end{array}$ & Contaminados & $\begin{array}{l}\text { Doble } \\
\text { ingreso }\end{array}$ & $\begin{array}{c}<20 \\
\text { colonias }\end{array}$ & $\begin{array}{l}<15 \\
\text { años }\end{array}$ & MINT & Negativos & & \\
\hline Amazonas & 9 & 29 & & 1 & & & & & 6 & 22 & 244,4 \\
\hline Chocó & 16 & 33 & 1 & 4 & & 1 & & & 2 & 25 & 156,3 \\
\hline Caldas & 28 & 51 & & 9 & 2 & & & & 7 & 33 & 117,9 \\
\hline Casanare & 14 & 25 & 2 & 1 & & 2 & & & 4 & 16 & 114,3 \\
\hline Risaralda & 24 & 38 & & 4 & & 3 & & & 5 & 26 & 108,3 \\
\hline Cauca & 47 & 65 & & 1 & & 5 & & & 10 & 49 & 104,3 \\
\hline Boyacá & 14 & 17 & & 2 & & & & & 1 & 14 & 100,0 \\
\hline Santander & 52 & 75 & & 11 & & 5 & & & 6 & 51 & 98,1 \\
\hline Cesar & 37 & 47 & & 4 & & 2 & 1 & & 4 & 36 & 97,3 \\
\hline Caquetá & 20 & 22 & & 1 & & & & & 3 & 18 & 90,0 \\
\hline Nariño & 34 & 40 & & 5 & & 2 & & & 8 & 25 & 73,5 \\
\hline Magdalena & 24 & 14 & & 1 & & & & & & 13 & 54,2 \\
\hline Bogotá & 87 & 63 & & 7 & & 4 & & & 7 & 45 & 51,7 \\
\hline Antioquia & 248 & 149 & & 3 & & 13 & & & 14 & 115 & 46,4 \\
\hline Bolívar & 44 & 32 & & 4 & & 1 & & & 7 & 18 & 40,9 \\
\hline Subtotal & 698 & 700 & 11 & 58 & 2 & 38 & 1 & 0 & 84 & 506 & 72,5 \\
\hline Vichada & 2 & 4 & & & & & & & & 4 & 200,0 \\
\hline Atlántico & 71 & 100 & & 1 & & 6 & & & 3 & 90 & 126,8 \\
\hline Quindío & 25 & 38 & & 2 & & 2 & & & 3 & 31 & 124,0 \\
\hline Valle & 101 & 164 & 2 & 11 & 2 & 12 & 1 & & 18 & 118 & 116,8 \\
\hline Sucre & 10 & 11 & & & & & & & & 11 & 110,0 \\
\hline Guaviare & 8 & 11 & & 1 & & & & & 2 & 8 & 100,0 \\
\hline Meta & 40 & 41 & & & & & 1 & 1 & 3 & 36 & 90,0 \\
\hline Putumayo & 27 & 23 & & 5 & 1 & & & & 2 & 15 & 55,6 \\
\hline San Andrés Islas & 2 & 4 & 1 & & & 1 & & & 1 & 1 & 50,0 \\
\hline Arauca & 14 & 18 & 1 & 7 & 1 & 1 & & & 1 & 7 & 50,0 \\
\hline Norte de Santander & 68 & 37 & & & & 2 & & & 3 & 32 & 47,1 \\
\hline Tolima & 40 & 24 & & & 1 & 1 & & & 4 & 18 & 45,0 \\
\hline Cundinamarca & 16 & 12 & & 1 & & 1 & & & 4 & 6 & 37,5 \\
\hline La Guajira & 65 & 33 & & 3 & & 1 & & & 5 & 24 & 36,9 \\
\hline Guainía & 4 & 3 & & & & 1 & & & 1 & 1 & 25,0 \\
\hline Córdoba & 44 & 15 & & 2 & & 1 & & & 1 & 11 & 25,0 \\
\hline Huila & 29 & 11 & & & & & & & 5 & 6 & 20,7 \\
\hline Vaupés & 2 & 1 & & & & & & & 1 & 0 & 0,0 \\
\hline Subtotal & 568 & 550 & 4 & 33 & 5 & 29 & 2 & 1 & 57 & 419 & 73,8 \\
\hline Total & 1266 & 1250 & 15 & 91 & 7 & 67 & 3 & 1 & 141 & 925 & 73,1 \\
\hline
\end{tabular}

Bajo recuento: cultivos procesados para pruebas de susceptibilidad con recuentos en controles de crecimiento menores de 200 unidades formadoras de colonia.

Contaminados: cultivos con crecimiento de bacilos ácido-alcohol resistentes contaminados con microorganismos ácido-alcohol sensibles

Doble ingreso: cultivos de un mismo paciente procedentes de dos laboratorios de salud pública diferentes.

$<20$ colonias: cultivos recibidos con crecimiento menor de 20 colonias, los cuales no fueron procesados para prueba de susceptibilidad.

$<15$ años: pacientes con tuberculosis infantil, no ingresaron al estudio por criterios de exclusión.

MNT: cultivos con aislamiento de micobacterias no tuberculosas (MNT).

Negativos: cultivos de pacientes negativos que ingresaron al estudio con baciloscopia positiva.

Este proyecto recibió el aval del Comité de Ética del Instituto Nacional de Salud.

\section{Resultados}

Casos nuevos. La prevalencia de resistencia global de M. tuberculosis en los $925(73,1 \%)$ cultivos provenientes de pacientes no tratados incluidos en el estudio fue de $11,78 \%$ (IC 95\%: 9,86-14,02) y la de MDR-TB, 2,38\% (IC 95\%: $1,58-3,57)$.
Las cepas de MDR-TB que presentaron resistencia combinada con estreptomicina más etambutol, es decir, resistentes a los cuatro fármacos utilizados en el tratamiento acortado supervisado, fueron 12 $(1,30 \%)$. Las cepas MDR-TB presentaron todas las combinaciones variables de resistencia a isoniacida más rifampicina, excepto isoniacida más rifampicina más etambutol. No se encontraron combinaciones de resistencia a etambutol más estreptomicina. 
En este grupo de pacientes, la mayor resistencia de $M$. tuberculosis a un solo medicamento se encontró para la estreptomicina $(4,32 \%)$, seguida de la isoniacida (2,27\%); no se encontró resistencia solamente a rifampicina ni a etambutol.

De igual forma, las combinaciones de isoniacida más rifampicina más etambutol, y rifampicina más etambutol, rifampicina más estreptomicina y rifampicina más estreptomicina más etambutol no presentaron resistencia (cuadro 2).

Casos previamente tratados. Se recibieron 264 cultivos de pacientes previamente tratados cuyas condiciones de ingreso al estudio fueron: 110 $(41,7 \%)$ abandonos, $72(27,3 \%)$ recaídas, 60 $(22,7 \%)$ fracasos, $17(6,4 \%)$ tratamientos terminados y $5(1,9 \%)$ pacientes, de los cuales no se obtuvo información con respecto al tratamiento anterior. La prevalencia de resistencia global fue de 44,32\% (IC 95\%: 38,45-50,35) y la de MDR-TB fue de 31,44\% (IC 95\%: 26,14-37,27).

En este grupo, la mayor resistencia de $M$. tuberculosis a uno de los fármacos antituberculosos se encontró para la estreptomicina $(5,68 \%)$, seguida de isoniacida $(1,89 \%)$ y rifampicina $(0,38 \%)$; no se encontró resistencia únicamente al etambutol.

Las cepas con MDR-TB que presentaron todas las combinaciones posibles de resistencia fueron $32(12,12 \%)$ resistentes a los cuatro fármacos utilizados en el tratamiento acortado supervisado. Las cepas resistentes a la isoniacida presentaron todas las combinaciones posibles de resistencia, mientras que las combinaciones de resistencia con rifampicina sólo se presentaron con etambutol (cuadro 3).

La distribución de la MDR-TB en el grupo de pacientes previamente tratados fue de $50,6 \%$ en los fracasos, $25,3 \%$ en los abandonos y $19,3 \%$ en las recaídas.

Control de calidad. En el control de calidad hecho a las baciloscopias de diagnóstico se obtuvo un porcentaje de concordancia del 99,9\%. Para el control de calidad de los medios de cultivo empleados para el diagnóstico y las pruebas de susceptibilidad, se recibieron 70 lotes preparados por los laboratorios de salud pública y el
Cuadro 2. Prevalencia de la resistencia a los fármacos antituberculosos en casos no tratados, Colombia 20042005.

\begin{tabular}{|c|c|c|c|}
\hline \multirow[t]{2}{*}{ Colombia } & \multicolumn{3}{|c|}{ Casos no tratados } \\
\hline & $\mathbf{n}$ & $\%$ & IC $95 \%$ \\
\hline Total de pruebas & 925 & & \\
\hline Susceptibilidad total & 816 & 88,22 & $85,98-90,14$ \\
\hline Resistencia total & 109 & 11,78 & $9,86-14,02$ \\
\hline \multicolumn{4}{|c|}{ Resistencia a un fármaco } \\
\hline Isoniacida $(\mathrm{H})$ & 21 & 2,27 & $1,49-3,45$ \\
\hline Rifampicina (R) & 0 & 0,00 & $0,00-0,41$ \\
\hline Etambutol (E) & 0 & 0,00 & $0,00-0,41$ \\
\hline Estreptomicina (S) & 40 & 4,32 & $3,19-5,83$ \\
\hline \multicolumn{4}{|c|}{ Resistencia a H más $R$ y a otro medicamento } \\
\hline H más $\mathrm{R}$ & 3 & 0,32 & $0,11-0,95$ \\
\hline $\mathrm{H}$ más $\mathrm{R}$ más $\mathrm{E}$ & 0 & 0,00 & $0,00-0,41$ \\
\hline $\mathrm{H}$ más $\mathrm{R}$ más $\mathrm{S}$ & 7 & 0,76 & $0,37-1,55$ \\
\hline H más $R$ más $S$ más $E$ & 12 & 1,30 & $0,74-2,25$ \\
\hline \multicolumn{4}{|c|}{ Resistencia a $\mathrm{H}$ y a otro medicamento } \\
\hline$H$ más $E$ & 2 & 0,22 & $0,06-0,78$ \\
\hline $\mathrm{H}$ más S & 21 & 2,27 & $1,49-3,45$ \\
\hline$H$ más $E$ más $S$ & 3 & 0,32 & $0,11-0,95$ \\
\hline \multicolumn{4}{|c|}{ Resistencia a $\mathbf{R}$ y a otro medicamento } \\
\hline $\mathrm{R}$ más $\mathrm{E}$ & 0 & 0,00 & $0,00-0,41$ \\
\hline $\mathrm{R}$ más $\mathrm{S}$ & 0 & 0,00 & $0,00-0,41$ \\
\hline$R$ más $E$ más $S$ & 0 & 0,00 & $0,00-0,41$ \\
\hline Otra resistencia & & & \\
\hline E más S & 0 & 0,00 & $0,00-0,41$ \\
\hline \multicolumn{4}{|c|}{ Total de resistencia por medicamento } \\
\hline Resistencia a H & 69 & 7,46 & $5,94-9,33$ \\
\hline Resistencia a R & 22 & 2,38 & $1,58-3,57$ \\
\hline Resistencia a E & 17 & 1,84 & $1,15-2,92$ \\
\hline Resistencia a S & 83 & 8,97 & $7,30-10,99$ \\
\hline \multicolumn{4}{|c|}{ Multirresistencia } \\
\hline MDR-TB & 22 & 2,38 & $1,58-3,57$ \\
\hline
\end{tabular}

Instituto Nacional de Salud; 66 (94\%) cumplieron con los parámetros para realizar el diagnóstico de M. tuberculosis, así como las pruebas de susceptibilidad.

Los resultados del control de calidad directo realizado por el laboratorio supranacional a las pruebas de susceptibilidad de cada uno de los fármacos presentó para la estreptomicina una sensibilidad de 100\% (IC 95\%: 95,45-100), especificidad de 86,67\% (IC 95\%: 83,23-90,10); para la isoniacida, una sensibilidad de $100 \%$ (IC 95\%: 98,88-100) y especificidad de 100\% (IC 95\%: 96,15-100); para la rifampicina, una sensibilidad de 100\% (IC 95\%: 96,67-100) y especificidad de 
Cuadro 3. Prevalencia de la resistencia a los fármacos antituberculosos en casos previamente tratados, Colombia 2004-2005.

\begin{tabular}{|c|c|c|c|}
\hline \multirow[t]{2}{*}{ Colombia } & \multicolumn{3}{|c|}{ Casos previamente tratados } \\
\hline & $\mathbf{n}$ & $\%$ & IC $95 \%$ \\
\hline Total de pruebas & 264 & & \\
\hline Susceptibilidad total & 147 & 55,68 & $49,65-61,55$ \\
\hline Resistencia total & 117 & 44,32 & $38,45-50,35$ \\
\hline \multicolumn{4}{|c|}{ Resistencia a un medicamento } \\
\hline Isoniacida $(\mathrm{H})$ & 5 & 1,89 & $0,81-4,36$ \\
\hline Rifampicina $(\mathrm{R})$ & 1 & 0,38 & $0,07-2,11$ \\
\hline Etambutol (E) & 0 & 0,00 & $0,00-1,43$ \\
\hline Estreptomicina (S) & 15 & 5,68 & $3,47-9,16$ \\
\hline \multicolumn{4}{|c|}{ Resistencia a $\mathrm{H}$ más $\mathrm{R}$ y a otro medicamento } \\
\hline $\mathrm{H}$ más $\mathrm{R}$ & 9 & 3,41 & $1,80-6,35$ \\
\hline $\mathrm{H}$ más $\mathrm{R}$ más $\mathrm{E}$ & 5 & 1,89 & $0,81-4,36$ \\
\hline $\mathrm{H}$ más $\mathrm{R}$ más $\mathrm{S}$ & 37 & 14,02 & $10,34-18,72$ \\
\hline$H$ más $R$ más $S$ más $E$ & 32 & 12,12 & $8,72-16,61$ \\
\hline \multicolumn{4}{|c|}{ Resistencia a $\mathrm{H}$ y a otro medicamento } \\
\hline $\mathrm{H}$ más $\mathrm{E}$ & 2 & 0,76 & $0,21-2,72$ \\
\hline $\mathrm{H}$ más $\mathrm{S}$ & 8 & 3,03 & $1,54-5,86$ \\
\hline $\mathrm{H}$ más $\mathrm{E}$ más $\mathrm{S}$ & 2 & 0,76 & $0,21-2,72$ \\
\hline \multicolumn{4}{|c|}{ Resistencia a $\mathbf{R}$ y a otro medicamento } \\
\hline $\mathrm{R}$ más $\mathrm{E}$ & 1 & 0,38 & $0,07-2,11$ \\
\hline $\mathrm{R}$ más $\mathrm{S}$ & 0 & 0,00 & $0,00-1,43$ \\
\hline$R$ más $E$ más $S$ & 0 & 0,00 & $0,00-1,43$ \\
\hline Otra resistencia & & & \\
\hline E más S & 0 & 0,00 & $0,00-1,43$ \\
\hline \multicolumn{4}{|c|}{ Total de resistencia por medicamento } \\
\hline Resistencia a H & 100 & 37,88 & $32,24-43,86$ \\
\hline Resistencia a R & 85 & 32,20 & $26,85-38,05$ \\
\hline Resistencia a E & 42 & 15,91 & $11,99-20,81$ \\
\hline Resistencia a S & 94 & 35,61 & $30,07-41,55$ \\
\hline \multicolumn{4}{|c|}{ Multirresistencia } \\
\hline MDR-TB & 83 & 31,44 & $26,14-37,27$ \\
\hline
\end{tabular}

100\% (IC 95\%: 96,47-100), y para el etambutol, una sensibilidad de 95,24\% (IC 95\%: 92,80-97,67) y una especificidad de 100\% (IC 95\%: 97,75-100). Los resultados del control de calidad indirecto presentaron para la estreptomicina una sensibilidad de 97,72\% (IC 95\%: 92,99-99,41) y especificidad de $82,08 \%$ (IC 95\%: 70,41-90,01); para la isoniacida, una sensibilidad de $99,18 \%$ (IC 95\%: $94,88-99,95$ ) y una especificidad de $88,15 \%$ (IC 95\%: 78,82-94,10); para la rifampicina, una sensibilidad de 100\% (IC 95\%: 93,60-100) y una especificidad de 92,96\% (IC 95\%: 86,69-96,56), y para el etambutol, sensibilidad de 100\% (IC 95\%: $87,35-100$ ) y especificidad de $92,12 \%$ (IC 95\%: $86,62-95,56)$.

\section{Discusión}

La vigilancia que se llevó a cabo permitió conocer la magnitud de la resistencia de $M$. tuberculosis a los fármacos antituberculosos en los casos no tratados y previamente tratados. Los resultados de este estudio demuestran que la resistencia y la MDR-TB en el país presentan una ligera tendencia al aumento con respecto a los estudios anteriores, aunque no significativa desde el punto de vista estadístico. El cálculo del tamaño de la muestra que se planificó es representativo para el país.

Los datos de resistencia no son significativos para evaluar la tendencia a nivel departamental. El tamaño estimado de la muestra para el país fue de 1.266 pacientes; se logró recolectar 1.250 (98,7\%) muestras, de las cuales, 925 (73,1\%) resultaron aptas para el estudio. Esto no afecta la representatividad de la muestra, toda vez que la precisión deseada pasa del $0,5 \%$ al $0,54 \%$. Sin embargo, no todos los departamentos aportaron las muestras en las cantidades requeridas, lo cual introduce un sesgo de selección para la muestra nacional.

Para el grupo de pacientes no tratados, la mayor resistencia de $M$. tuberculosis a uno de los fármacos antituberculosos, se encontró con la estreptomicina $(4,32 \%)$, seguida de la isoniacida $(2,27 \%)$.

En el estudio nacional del año 2000 los valores encontrados para estos fármacos fueron de 5,6\% y $3,4 \%$, respectivamente, por lo cual no existen diferencias estadísticamente significativas. No se encontró resistencia solamente a rifampicina ni a etambutol en este estudio, similar a lo encontrado en el estudio del año 2000 (5).

La MDR-TB en los casos previamente tratados se presentó en $50,6 \%$ de los fracasos, lo cual indica que se debe fortalecer la vigilancia mediante el cultivo y las pruebas de susceptibilidad ante cualquier sospecha de fracaso del tratamiento, pues para estos pacientes se requieren esquemas individuales de tratamiento y de manejo en un centro de atención de alto nivel de complejidad. Por otro lado, la MDR-TB en los abandonos fue de $25,3 \%$ y en las recaídas de $19,3 \%$, lo que demuestra que el esquema regular estandarizado se debe mantener para la mayoría de los casos, 
así como la realización del cultivo y pruebas de susceptibilidad a los fármacos antituberculosos.

El alto porcentaje de abandonos como condición de ingreso en el grupo de pacientes previamente tratados indica que hacen falta estrategias que faciliten el cumplimiento de los pacientes del tratamiento, para mejorar las metas de curación del Programa Nacional de Control de Tuberculosis.

A nivel mundial, la MDR-TB muestra una alta prevalencia en varias áreas de la Federación Rusa y en otros países que conformaban la antigua Unión Soviética (Kazakistán, Uzbekistán, Estonia, Letonia y Lituania), como también en algunas áreas fuera de Europa, inclusive Israel y las provincias chinas de Liaoning y Henan. En la región de las Américas, Colombia ocupa el quinto lugar en prevalencia de la MDR-TB en casos no tratados para los años 1999 a 2002 con una tasa de $1,5 \%$, detrás de Ecuador (6,6\%), Puerto Rico $(2,0 \%)$, Argentina $(1,8 \%)$ y Honduras $(1,8 \%)(14)$.

En este estudio no se analizó la información de variables como infección asociada de tuberculosis$\mathrm{VIH} /$ sida, régimen de afiliación al sistema general seguridad social en salud y el aporte de casos por municipio.

La sensibilidad a los fármacos evaluados en las cepas de $M$. tuberculosis que están circulando en el país, indica que el esquema que proporciona actualmente el Programa Nacional de Control de Tuberculosis y establecido en la Guía de Atención Integral de la Tuberculosis (15) es adecuado para el tratamiento de los casos de tuberculosis.

Aunque la sensibilidad a los fármacos antituberculosos y la MDR-TB entre los casos no tratados en los dos últimos estudios nacionales no presentan variaciones estadísticas, es importante continuar con la expansión de la estrategia DOTS (Directly Observed Treatment Estrategy) o TAES (Tratamiento Acortado Estrictamente Supervisado) como mecanismo para fortalecer el Programa Nacional de Control de Tuberculosis y disminuir la prevalencia de la resistencia a los medicamentos en el país, además de realizar en forma permanente los análisis de cohortes de los pacientes con tuberculosis pulmonar en tratamiento $(16,17)$.
Con los anteriores resultados y conociendo las debilidades que el país presenta en el manejo de pacientes con MDR-TB, se debe fortalecer la vigilancia en aquellos departamentos con más casos, ejercer vigilancia y dirigir acciones para conseguir la curación del paciente.

\section{Agradecimientos}

A los profesionales responsables del Programa Nacional de Control de Tuberculosis y de los Laboratorios de Salud Pública de las secretarías de salud departamentales y de los distritos y las instituciones de salud del país.

Al Ministerio de la Protección Social y la Organización Panamericana de Salud en Colombia, por su acompañamiento y por los recursos para la financiación del estudio.

\section{A la Deutsche Lepra und Tuberkulosehilfe(DAHW)} por su colaboración.

A todo el personal del Laboratorio de Micobacterias por su compromiso durante la realización del estudio.

\section{Conflicto de intereses}

Los autores del artículo hacen constar que no existe, de manera directa o indirecta, ningún tipo de conflicto de intereses financieros, académicos o personales que puedan poner en peligro la validez de lo comunicado.

\section{Financiación}

Este trabajo fue financiado por OPS/OMS, el Ministerio de la Protección Social, el Instituto Nacional de Salud, las Secretarías de Salud departamentales y distritales y los Laboratorios Departamentales de Salud Pública del país.

\section{Referencias}

1. World Health Organization. Global tuberculosis control - surveillance, planning, financing. Geneve: World Health Organization; 2007. p. 376.

2. World Health Organization. Directrices para el tratamiento programático de la tuberculosis drogorresistente. Geneve: World Health Organization; 2006. p. 361.

3. Ministerio de la Protección Social, Organización Panamericana de la Salud. Situación de Salud en Colombia. Indicadores básicos. Bogotá: Ministerio de la Protección Social; 2006. 
4. Orozco LC, Aparicio G, Quintero O, Giraldo E, Ulloa I, León Cl. Resistencia de M. tuberculosis a los fármacos. Biomédica. 1981;1:130-4.

5. León $\mathrm{Cl}$, Sierra C, Naranjo N, Garzón MC, Guerrero MI. Segundo estudio nacional de resistencia primaria del $M$. tuberculosis a las drogas antituberculosas en Colombia. Infectio. 2002;6:83.

6. World Health Organization/International Union Against Tuberculosis and Lung Disease. Global project on antituberculosis drug resistance surveillance. Guidelines for surveillance of drug resistance in tuberculosis. Geneve: World Health Organization; 2002.

7. Canetti G, Rist N, Grosset J. Medida de la sensibilidad del bacilo tuberculoso a las drogas antibacilares por el método de las proporciones. Buenos Aires: Dirección Lucha Antituberculosa; 1965.

8. Canetti G, Wallace F, Khomenko A, Mahler HT, Menon NK, Rist N, et al. Advances in techniques of testing mycobacterial drug sensitivity and the use of sensitivity tests in tuberculosis control programs. Bull WHO. 1969;41:21-43.

9. Garzón M, Naranjo N, Sierra C, Llerena C, Orjuela D. Bacteriología del M. tuberculosis y micobacterias no tuberculosas. Manual de procedimientos. Bogotá: Instituto Nacional de Salud; 2002.

10. Orozco LC, León CI, Giraldo de BE, Quintero de RO, Ulloa de MI. El cultivo de esputo para el diagnóstico de la tuberculosis pulmonar. Biomédica. 1985;5:24-6.
11. Kudoh S, Kudoh A. A simple technique for culturing tubercle bacilli. Bull WHO. 1974;51:71-84.

12. Laszlo A, Rahman M, Raviglione M, Bustreo F. Quality assurance programme for drug susceptibility testing of Mycobacterium tuberculosis in the WHO/ IUATLD Supranational Laboratory Network: first round of proficiency testing. Int Tuberc Lung Dis. 1997;1:231-8.

13. World Health Organization. Global tuberculosis programme. Surveillance of drug resistance in tuberculosis. A user's guide to the software: SDRTB 4.0. Geneve: World Health Organization; 1996. p. 2.

14. World Health Organization. Anti-tuberculosis drug resistance in the world. Report No. 3. Geneve: World Health Organization; 2004.

15. Dirección General de Salud. Ministerio de Salud, Colombia. Normas técnicas y guías de atención. Resolución 00412 febrero 25 de 2000. Guías de Atención de la Tuberculosis pulmonar y extrapulmonar. Bogotá: Ministerio de Salud; 2000. p. 1-44.

16. Organización Mundial de la Salud. Plan mundial para detener la tuberculosis 2006-2015. Ginebra: Organización Mundial de la Salud; 2006.

17. Organización Panamericana de la Salud, Programas Nacionales de Control de la Tuberculosis, Ministerios de Salud Pública. Plan Regional de Tuberculosis 2006-2015. Washington D.C.: PAHO; 2006. 